[Radiocarbon, Vol. 19, No. 1, 1977, P. 12-18]

\title{
LABORATORY OF COSMOCHEMISTRY RADIOCARBON MEASUREMENTS I
}

\section{A K LAVRUKHINA and $\mathrm{V}$ A ALEXEEV}

V I Vernadsky Institute of Geochemistry and Analytical Chemistry, USSR Academy of Sciences, Moscow, USSR

The ${ }^{14} \mathrm{C}$ dates listed in this report on archaeologic and geologic samples were obtained mainly from 1969 to 1974. Pretreatment of wood charcoal and carbonized fossil soil by boiling in acid, alkali, and acid, again, is standard procedure. The method employed is liquid scintillation counting of synthesized benzene using the basic techniques described by Arslanov and Gromova (1968) converting sample $\rightarrow \mathrm{Li}_{2} \mathrm{C}_{2} \rightarrow \mathrm{C}_{2} \mathrm{H}_{2} \rightarrow$ $\mathrm{C}_{6} \mathrm{H}_{6}$ with an overall yield to ca $90 \%$. A vanadium-alumina catalyst is used at reduced temperature for the acetylene to benzene conversion. Anthracite coal was used for the dead carbon run.

Within the experimental error a one-to-one correlation between the activity of background and that of anthracite coal was obtained on two different runs. Several months of analyzing standards, backgrounds, and samples of known age reveal a high degree of precision and reliability in all systems (Alexeev et al, 1975). All measurements of ${ }^{14} \mathrm{C}$ activity of synthesized benzene were made on a liquid scintillation counter. The scintillator vial was viewed by two photomultipliers working in coincidence. The background counting rate was reduced by massive screening and by pulse-height selection. The counter is shielded by 20 to $30 \mathrm{~cm}$ of selected iron, 10 to $15 \mathrm{~cm}$ paraffin wax containing $25 \%$ boric acid, $10 \mathrm{~cm}$ stainless steel, and $2.5 \mathrm{~cm}$ mercury. The total weight is ca 50 tons (2 secs). Table 1 gives the pertinent information for the counter.

TABLe 1

\begin{tabular}{cccc}
\hline $\begin{array}{c}\text { Volume of } \\
\text { the counting } \\
\text { vial, } \mathrm{ml}\end{array}$ & $\begin{array}{c}\text { Background B, } \\
\mathrm{cpm}\end{array}$ & $\begin{array}{c}\text { Modern carbon } \\
\text { A, cpm }\end{array}$ & $\begin{array}{c}\text { Fig of } \\
\text { merit A/ } \sqrt{\mathbf{B}}\end{array}$ \\
\hline 5.5 & $3.98 \pm 0.02$ & $33.11 \pm 0.04$ & 16.6 \\
13.6 & $5.2 \pm 0.5$ & $87 \quad \pm 2$ & 38.5 \\
27.1 & $9.17 \pm 0.03$ & $180.0 \pm 0.3$ & 59.5 \\
56 & $14.5 \pm 0.5$ & $314 \quad \pm 3$ & 82.5 \\
\hline
\end{tabular}

All samples were measured at least twice, with an interval of up to one month between them. Dates are reported in conventional radiocarbon years, using the 5570 half-life, with 1950 as the reference year. The modern reference standard is $95 \%$ of the measured activity of the NBS oxalic acid standard. Age limits quoted are derived from $1 \sigma$ counting statistics of background, modern, and sample counts.

$\delta^{13} \mathrm{C}$ measurements are made on $\mathrm{CO}_{2}$ produced in charcoal or synthesized benzene combustion, and results are given relative to the PDB 
standard. The present-day value of $\Delta$ for each sample was calculated from the measured isotopic ratios using Broecker's formula (Broecker and Olson, 1961):

$$
\Delta=\delta^{14} \mathrm{C}-\left(2 \delta^{13} \mathrm{C}+50\right)\left(1+0.001 \delta^{14} \mathrm{C}\right)
$$

ACKNOWLEDGMENTS

We wish to thank collectors and submitters of the samples. Our special gratitude goes to E M Galimov, and his staff for the ${ }^{13} \mathrm{C} /{ }^{12} \mathrm{C}$ measurements. Pretreatments, benzene syntheses, and routine operation of the dating apparatus were carried out by $\mathrm{Z} \mathrm{K}$ Milnikova and $\mathrm{I} \mathrm{V}$ Smirnov, to whom we are much indebted.

\section{Ukrainian series}

\section{ARCHAEOLOGIC SAMPLES}

Wood from ancient tombs, Ukrainian SSR, USSR, coll and subm 1971 by V P Zolotun, A D Tsuryupa Agric Inst, Kherson, Ukrainian SSR.

\section{CSM-67. Ukrainian 1}

$4220 \pm 50$

Wood from depth $300 \mathrm{~cm}$, Tomb $1,10 \mathrm{~km}$ W of town Novaya Odessa of the Nikolaevskaya prov. Estimated age: 2600 to 2300 BC. Tripolskaya culture.

CSM-68. Ukrainian 2

Wood from depth $70 \mathrm{~cm}$, Tomb 4. Estimated age: 1000 to 800 BC. Kemmerijskaya culture.

\section{CSM-69. Ukrainian 3}

Wood from depth $250 \mathrm{~cm}$, Tomb 31 near village Borisivka, Odessa prov. Estimated age: 1150 to 850 BC. Culture of corpses burn.

CSM-70. Ukrainian 4

$4150 \pm 50$

Wood from Tomb 10, near village Majaki, Odessa prov. Estimated age is ca 2200 BC. Usatovskaya culture.

CSM-71. Ukrainian 5

Wood from depth $350 \mathrm{~cm}$, Tomb 6 near village Tatarbunary, Odessa prov. Estimated age: 1200 to 1000 BC. Srubnaya culture.

CSM-72. Ukrainian 6

$4390 \pm 60$

Wood from Tomb 27 near village Lyubimovka, Kherson prov. Estimated age: 2800 to $2500 \mathrm{BC}$.

\section{Siberian series}

\section{GEOLOGIC SAMPLES}

Samples are from a $14 \mathrm{~m}$ terrace of $\mathrm{R}$ Irkineeva, tributary of $\mathrm{R}$ Angara, near village Bedoba, RSFSR, USSR. Coll and subm 1969 by S A Laukhin, All-Union Scientific-Research Inst Min Prod, Moscow, USSR. 
CSM-10. Siberian 1

Wood, 6m below terrace surface.

CSM-11. Siberian 2

Wood, $8 \mathrm{~m}$ below terrace surface.

CSM-42. Siberian 3

Wood, $8.5 \mathrm{~m}$ below terrace surface. No pretreatment.

CSM-41. Siberian 4

Wood from same location as CSM-42. Sample was pretreated by boiling with $5 \% \mathrm{HCl}$ for 20 min, boiling with $2 \% \mathrm{NaOH}$ for $12 \mathrm{hr}$ with extracted humic acids, boiling with $5 \% \mathrm{HCl}$ for $30 \mathrm{~min}$. Between the stages the sample was washed with distilled water.

CSM-43. Siberian 5

$38,300 \pm 1000$

Humic acids extracted during pretreatment of sample CSM-41.

\section{Onikotan Island series}

Samples from Onikotan I near near Krinitsyno volcano, Kuril Is, USSR. Coll and subm 1969 by L D Sulerzhitsky, Inst Geol, Acad Sci USSR.

CSM-438(g)-I. Onikotan I. 1-1, 31 to $40 \mathrm{yr}$

$7650 \pm 70$

Completely carbonized tree trunk with a well pronounced trunk surface (bark has been preserved). Tree age is ca $40 \mathrm{yr}$. It died during eruption of Krinitsyno volcano. Layer of eruptive rocks is ca $40 \mathrm{~m}$ over sampling site. Four groups of tree rings, ca $10 \mathrm{yr}$ each, were prepared. CSM-438(g)-I was the group of outer tree rings, the last ca $10 \mathrm{yr}$ of tree growth.

$\begin{array}{llr}\text { CSM-438(g)-II. } & \text { Onikotan I. } 1-2,21 \text { to } 30 \mathrm{yr} & 7750 \pm 90 \\ \text { CSM-438(g)-III. } & \text { Onikotan I. } 1-3,11 \text { to } 20 \mathrm{yr} & 7500 \pm 80 \\ \text { CSM-438(g)-IV. Onikotan I. } 1-4,1 \text { to } 10 \mathrm{yr} & 7700 \pm 110 \\ \text { CSM-439(g). Onikotan I. } 2 & 7950 \pm 70\end{array}$

Carbonized fossil soil, $20 \mathrm{~cm}$ below CSM-438(g)-I.

$\operatorname{CSM-440}(\mathrm{g})$. Onikotan I. 3

$8230 \pm 90$

Carbonized fossil soil, $40 \mathrm{~cm}$ below CSM-438(g)-I.

CSM-440 (g) -G. Onikotan I. 4

$\mathbf{8 4 8 0} \pm \mathbf{5 0}$

Humic acids from sample CSM-440(g).

CSM-442(g)-I. Onikotan I. 5-1, 21 to 25 yr $9430 \pm 70$

Tree trunk, $2 \mathrm{~m}$ below CSM-438(g)-I. The tree died in a mud-ash flow. Five groups of tree rings, at 5 yr each, were prepared. CSM-442(g)-I was group of outer tree rings. 
CSM-442(g)-II. Onikotan I. 5-2, 16 to 20 yr $9770 \pm 100$

CSM-442(g)-III. Onikotan I. 5-3, 11 to 15 yr $9280 \pm 60$

CSM-442(g)-IV. Onikotan I. 5-4, 6 to $10 \mathrm{yr} \quad 9800 \pm 140$

$\operatorname{CSM-442}(\mathrm{g})-\mathrm{V}$. Onikotan I. 5-5, 1 to 5 yr $\quad 9330 \pm 120$

$\operatorname{CSM-443}(\mathrm{g})$. Onikotan I. $6 \quad 24,500 \pm 740$

Carbonized soil at base of a thick stratum, ca $30 \mathrm{~m}$, of pumices formed by eruption of Nemo volcano.

\section{Simushir Island series}

Recent wood from foot of N slope of Uratman volcano, Simushir I., Kuril Is, USSR. Coll and subm 1969 by L D Sulerzhitsky, Inst Geol, Acad Sci USSR.

$\operatorname{CSM-453(g).~Simushir~I.~1,~}+20 \mathrm{~m}$

$$
\delta^{14} \mathrm{C}=17 \pm 4 \%
$$

$\operatorname{CSM-454}(\mathrm{g})$. Simushir I. 2, $+40 \mathrm{~m}$

$\delta^{14} \mathrm{C}=-\mathbf{4 1} \pm \mathbf{4} \%$

$\operatorname{CSM-455}(\mathrm{g})$. Simushir I. 3, +100m

$\delta^{14} \mathrm{C}=-\mathbf{3 4} \pm \mathbf{4} \%$

\section{Kamchatka Peninsula series}

Tree rings, alder, near Kozyrevsk village, Kamchatka R, Kamchatka peninsula, USSR. The tree was cut in 1967. Coll and subm 1969 by L D Sulerzhitsky, Inst Geol, Acad Sci USSR.

CSM-452(g)-O. Kamchatka Peninsula $1 \quad \delta^{14} \mathrm{C}=382 \pm 6 \%$ Tree rings, grown 1948 to 1967.

CSM-452(g) -A. Kamchatka Peninsula $2 \quad \delta^{14} \mathrm{C}=-37 \pm 5 \%$ Tree rings, grown 1943 to 1947.

CSM-452(g)-B. Kamchatka Peninsula $3 \quad \delta^{14} \mathrm{C}=-\mathbf{4 8} \pm \mathbf{5} \%$ o Tree rings, grown 1938 to 1942.

TABle 2

$\delta^{13} \mathrm{C}$ values

\begin{tabular}{lclc}
\hline Lab no. & Year* & Sample & $\delta^{13} \mathrm{C}, \% o( \pm 0.4 \% 0)$ \\
\hline CSM-46 & 1905 & Charcoal & -26.2 \\
CSM-46 & 1905 & Benzene & -26.1 \\
CSM-52 & 1908 & Benzene & -24.4 \\
CSM-55 & $1910-1911$ & Benzene & -25.9 \\
CSM-61 & $1913-1914$ & Benzene & -26.2 \\
CSM-65 & $1915-1916$ & Benzene & -26.4 \\
\hline & & Average & $-25.9 \pm 0.6$ \\
\hline
\end{tabular}

* Single yr correspond to spring-summer parts of rings, double yr to autumnwinter parts. 
TABLE 3

$\Delta$ data of sequoia tree rings

\begin{tabular}{|c|c|c|}
\hline Lab no. & Year* & $\Delta \pm \delta \Delta, \%$ \\
\hline CSM-81 & 1890 & $18 \pm 6$ \\
\hline CSM-82 & $1890-1891$ & $13 \pm 6$ \\
\hline CSM-83 & 1891 & $12 \pm 3$ \\
\hline CSM-84 & 1891-1892 & $14 \pm 3$ \\
\hline CSM-85 & 1892 & $13 \pm 3$ \\
\hline CSM-86 & 1892-1893 & $32 \pm 4$ \\
\hline CSM-87 & 1893 & $5 \pm 6$ \\
\hline CSM-88 & 1893-1894 & $8 \pm 7$ \\
\hline CSM-89 & 1894 & $27 \pm 4$ \\
\hline CSM-90 & 1894-1895 & $21 \pm 3$ \\
\hline CSM-91 & 1895 & $36 \pm 4$ \\
\hline CSM-92 & $1895-1896$ & $26 \pm 6$ \\
\hline CSM-93 & 1896 & $19 \pm 3$ \\
\hline CSM-94 & $1896-1897$ & $27 \pm 7$ \\
\hline CSM-95 & 1897 & $19 \pm 3$ \\
\hline CSM-96 & 1897-1898 & $23 \pm 3$ \\
\hline CSM-97 & 1898 & $27 \pm 3$ \\
\hline CSM-98 & 1898-1899 & $19 \pm 3$ \\
\hline CSM-99 & 1899 & $14 \pm 8$ \\
\hline CSM-100 & $1899-1900$ & $18 \pm 4$ \\
\hline CSM-101 & 1900-1901 & $27 \pm 4$ \\
\hline CSM-102 & 1901 & $16 \pm 3$ \\
\hline CSM-103 & 1901-1902 & $22 \pm 3$ \\
\hline CSM-104 & 1902 & $19 \pm 3$ \\
\hline CSM-105 & 1902-1903 & $24 \pm 3$ \\
\hline CSM-106 & 1903 & $16 \pm 3$ \\
\hline CSM-107 & 1903-1904 & $19 \pm 3$ \\
\hline CSM-108 & 1904 & $24 \pm 5$ \\
\hline CSM-45 & 1904-1905 & $15 \pm 3$ \\
\hline CSM-46 & 1905 & $15 \pm 3$ \\
\hline CSM-47 & 1905-1906 & $16 \pm 4$ \\
\hline CSM-48 & 1906 & $30 \pm 3$ \\
\hline CSM-49 & 1906-1907 & $25 \pm 5$ \\
\hline CSM-50 & 1907 & $30 \pm 6$ \\
\hline CSM-51 & 1907-1908 & $19 \pm 4$ \\
\hline CSM-52 & 1908 & $22 \pm 5$ \\
\hline CSM-53 & 1908-1909 & $30 \pm 3$ \\
\hline CSM-54 & $1909-1910$ & $-5 \pm 4$ \\
\hline CSM-55 & $1910-1911$ & $14 \pm 9$ \\
\hline CSM-56 & 1911 & $12 \pm 3$ \\
\hline CSM-57 & $1911-1912$ & $19 \pm 6$ \\
\hline
\end{tabular}


TABLE 3 (continued)

\begin{tabular}{llr}
\hline Lab no. & Year* & $\Delta \pm \delta \Delta, \%$ \\
\hline CSM-58 & 1912 & $16 \pm 4$ \\
CSM-59 & $1912-1913$ & $5 \pm 3$ \\
CSM-60 & 1913 & $2 \pm 3$ \\
CSM-61 & $1913-1914$ & $15 \pm 3$ \\
CSM-62 & 1914 & $2 \pm 3$ \\
CSM-63 & $1914-1915$ & $-41 \pm 4$ \\
CSM-64 & 1915 & $4 \pm 3$ \\
CSM-65 & $1915-1916$ & $-2 \pm 5$ \\
\hline
\end{tabular}

* See Table 2.

CSM-452(g) -C. Kamchatka Peninsula $4 \quad \delta^{14} \mathrm{C}=-47 \pm 8 \%$ Tree rings, grown 1933 to 1937.

CSM-452(g)-D. Kamchatka Peninsula $5 \quad \delta^{14} \mathrm{C}=-41 \pm 5 \%$ Tree rings, grown 1928 to 1932.

CSM-452(g)-E. Kamchatka Peninsula $6 \quad \delta^{14} \mathrm{C}=-\mathbf{4 7} \pm 6 \%$ Tree rings, grown 1923 to 1927.

CSM-452(g) -F. Kamchatka Peninsula $7 \quad \delta^{14} \mathrm{C}=-27 \pm 6 \%$ Tree rings, grown before 1923.

\section{Sequoia tree rings series}

Wood (sequoia) from Nikitsk Bot garden, Yalta, Crimea, USSR. Tree, ca 100 yr old, felled in 1964. Coll and subm 1971 by L D Sulerzhitsky, Inst Geol, Acad Sci USSR. Subsequently dendro-dated by LDS. The ${ }^{14} \mathrm{C}$ content was determined both in spring-summer and in autumnwinter parts of each tree ring. $\delta^{13} \mathrm{C}$ was determined for some samples by E M Galimov, I M Gubkin Inst Oil-Chemical \& Gas Industry, Moscow, USSR (present address: V I Vernadsky Inst Geochem \& Analytical Chem USSR Acad Sci, Moscow, USSR). $\delta^{13} \mathrm{C}$ data are shown in Table 2.

These $\delta^{13} \mathrm{C}$ values were used for determination of $\Delta$ values of the same samples. The average value of the $\delta^{13} \mathrm{C}(-25.9 \pm 0.6 \%)$ was used to determine $\Delta$ values of other samples. $\Delta$ data are shown in Table 3.

A positive correlation between ${ }^{14} \mathrm{C}$ contents and no. of sunspots was found (fig 1). Correlation coefficient $+0.57 \pm 0.12$ was obtained with phase shift ca $1.7 \mathrm{yr}$, which corresponds to reliability of conclusion about positive correlation by $>99 \%$. Obtained data discussed in detail previously (Alexeev et al, 1975; Lavrukhina et al, 1973). 


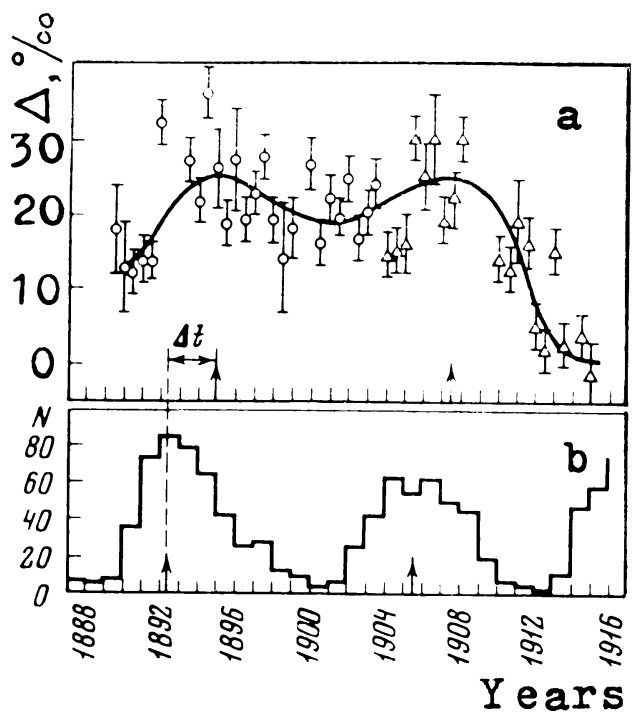

Fig. 1. Time variations of the ${ }^{14} \mathrm{C}$ content (a) and of sunspot numbers (b). The maxima are shown by arrows. The $\Delta t$ is ca $1.7 \mathrm{yr}$.

\section{REFERENCES}

Alexeev, V A, Lavrukhina, A K, and Milnikova, Z K, 1975, Variations of radiocarbon content in annual rings of the sequoia (1890-1916): Geokhimia, no. 5, p 667-675.

Arslanov, Kh A and Gromova, L I, 1968, Cyclic trimerization of acetylene and alkylacetylene on the chromalumosilicate catalyzer: Doklady Akad Nauk SSSR, v 183, p 881-884.

Broecker, W S and Olson, E A, 1961, Lamont radiocarbon measurements VIII: Radiocarbon, v 3, p 176-204.

Lavrukhina, A K, Alexeev, V A, Galimov, E M, and Sulerzhitsky, L D, 1973, Radiocarbon in the rings of sequoia: Doklady Akad Nauk SSSR, v 210, p 941-943. 